\title{
ON SELF-SIMILAR MEASURES WITH ABSOLUTELY CONTINUOUS PROJECTIONS AND DIMENSION CONSERVATION IN EACH DIRECTION
}

\author{
ARIEL RAPAPORT
}

\begin{abstract}
Relying on results due to Shmerkin and Solomyak, we show that outside a 0-dimensional set of parameters, for every planar homogeneous selfsimilar measure $\nu$, with strong separation, dense rotations and dimension greater than 1 , there exists $q>1$ such that $\left\{P_{z} \nu\right\}_{z \in S} \subset L^{q}(\mathbb{R})$. Here $S$ is the unit circle and $P_{z} w=\langle z, w\rangle$ for $w \in \mathbb{R}^{2}$. We then study such measures. For instance, we show that $\nu$ is dimension conserving in each direction and that the map $z \rightarrow P_{z} \nu$ is continuous with respect to the weak topology of $L^{q}(\mathbb{R})$.
\end{abstract}

\section{INTRODUCTION}

Denote by $\mathbb{D}$ the open unit disc in $\mathbb{C}$ and let $0 \neq \lambda \in \mathbb{D}$ be with $\arg \lambda \notin \pi \mathbb{Q}$. Consider a homogeneous IFS on $\mathbb{C}$

$$
\left\{\varphi_{i}(w)=\lambda w+a_{i}\right\}_{i \in \Lambda},
$$

with the strong separation condition (SSC), and a self-similar measure

$$
\nu=\sum_{i \in \Lambda} p_{i} \cdot \varphi_{i} \nu
$$

It is among the most basic planar self-similar measures. Hence it is a natural question in fractal geometry to study the dimension and continuity of the projections $\left\{P_{z} \nu\right\}_{z \in S}$ and slices

$$
\left\{\left\{\nu_{z, w}\right\}_{w \in \mathbb{C}}: z \in S\right\} .
$$

Here $S$ is the unit circle of $\mathbb{C}, P_{z} w=\operatorname{Re}(z \cdot \bar{w})$ for $w \in \mathbb{C}$, and $\left\{\nu_{z, w}\right\}_{w \in \mathbb{C}}$ is the disintegration of $\nu$ with respect to $P_{z}^{-1}(\mathcal{B})$, where $\mathcal{B}$ is the Borel $\sigma$-algebra.

Dimensionwise, the behaviour of the projections is as regular as possible. Indeed, Hochman and Shmerkin [HS] have proven that $P_{z} \nu$ is exact dimensional, with

$$
\operatorname{dim} P_{z} \nu=\min \{1, \operatorname{dim} \nu\},
$$

for each $z \in S$. A version of this, for self-similar sets with dense rotations, was first proven by Peres and Shmerkin [PS]. Considering the absolute continuity of the projections, Shmerkin and Solomyak [SS1] have shown, assuming $\operatorname{dim} \nu>1$, that the set

$$
E=\left\{z \in S: P_{z} \nu \text { is singular }\right\}
$$

has zero Hausdorff dimension.

Date: September 26, 2018.

2000 Mathematics Subject Classification. Primary: 28A80, Secondary: 28A78.

Key words and phrases. Self-similar sets and measures, absolute continuity, dimension conservation. 
Let us turn to discuss the concept of dimension conservation and the dimension of slices. A Borel probability measure $\mu$ on $\mathbb{C}$ is said to be dimension conserving (DC), with respect to the projection $P_{z}$, if

$$
\operatorname{dim}_{H} \mu=\operatorname{dim}_{H} P_{z} \mu+\operatorname{dim}_{H} \mu_{z, w} \text { for } \mu \text {-a.e. } w \in \mathbb{C},
$$

where $\operatorname{dim}_{H}$ stands for Hausdorff dimension. It always holds that $\mu$ is DC with respect to $P_{z}$ for almost every $z \in S$. This follows from results, valid for general measures, regarding the typical dimension of projections (see [HK]) and slices (see [JM]). Falconer and Jin FJ1 have shown that $\mu$ is DC, with respect to $P_{z}$ for all $z \in S$, whenever $\mu$ is self-similar with a finite rotation group. An analogues statement, for self-similar sets with the SSC, was first proven by Furstenberg [Fur]. Another related result for sets is due to Falconer and Jin [FJ2]. They showed that if $K \subset \mathbb{C}$ is self-similar, with $\operatorname{dim} K>1$ and a dense rotation group, then for every $\epsilon>0$ there exists $N_{\epsilon} \subset S$, with $\operatorname{dim}_{H} N_{\epsilon}=0$, such that for $z \in S \backslash N_{\epsilon}$ the set

$$
\left\{x \in \mathbb{R}: \operatorname{dim}_{H}\left(K \cap P_{z}^{-1}\{x\}\right)>\operatorname{dim} K-1-\epsilon\right\}
$$

has positive length.

Taking these results into account, one might expect the sets $E$, defined above, and

$$
F=\left\{z \in S: \nu \text { is not DC with respect to } P_{z}\right\}
$$

to be empty whenever the dimension of $\nu$ exceeds 1 . Nevertheless, in Rap2 the author has constructed an example, of a measure $\nu$ as above, for which $E$ and $F$ are nonempty and even residual. Moreover, in this example there exists a $G_{\delta}$-subset of directions $z \in S$ for which $\nu_{z, w}$ is discrete, and hence has dimension 0 , for $\nu$-a.e. $w \in \mathbb{C}$.

In this paper we shall show that, despite of the last result, it is typically the case that $E$ and $F$ are empty. More precisely, we shall prove the following theorem. A sharper version of it is stated in Section 3 .

Theorem. There exists a set $\mathcal{E} \subset \mathbb{D}$, with $\operatorname{dim}_{H} \mathcal{E}=0$, such that the following holds. Let $\lambda \in \mathbb{D} \backslash \mathcal{E}$ be with $\arg \lambda \notin \pi \mathbb{Q}$, let $\nu$ be a self-similar measure with the SSC as in (1.2), and assume that $\operatorname{dim}_{H} \nu>1$. Then there exists $1<q<\infty$ such that,

(1) $P_{z} \nu \in L^{q}(\mathbb{R})$ for each $z \in S$;

(2) the map which takes $z \in S$ to $P_{z} \nu \in L^{q}(\mathbb{R})$ is continuous with respect to the weak topology of $L^{q}(\mathbb{R})$;

(3) for each $z \in S$ the measure $\nu_{z, w}$ has exact dimension $\operatorname{dim}_{H} \nu-1$ for $\nu$-a.e. $w \in \mathbb{C}$.

Note that by parts (10) and (3) it follows that $\nu$ is DC, with respect to $P_{z}$, for all $z \in S$.

Part (1) follows almost directly from results which are due to Shmerkin and Solomyak [SS1] and [SS2] and Shmerkin [Sh1] and [Sh2]. Our main contribution is the derivation of parts (2) and (3). For this we first show that, assuming part (1), the collection $\left\{P_{z} \nu\right\}_{z \in S}$ is bounded in $\left(L^{q}(\mathbb{R}),\|\cdot\|_{q}\right)$. The reflexivity of $L^{q}(\mathbb{R})$, for $1<q<\infty$, plays an important role here.

We obtain a much stronger form of continuity in (2) if instead of $\operatorname{dim}_{H} \nu>1$ we assume that the correlation dimension of $\nu$ exceeds 1 . More precisely, in Theorem 3.7 it is shown that, under this stronger assumption, there exits $\gamma>0$ so that 
$P_{z} \nu$ lies in the 2, $\gamma$-Sobolev space for each $z \in S$ and that the map $z \rightarrow P_{z} \nu$ is continuous with respect to the corresponding Sobolev norm.

The boundedness of $\left\{P_{z} \nu\right\}_{z \in S}$ in $L^{q}(\mathbb{R})$ gives also a result regarding the measure class of the projections. Denote by $K$ the self-similar set corresponding to the IFS (1.1). By extending an argument given in [MS] and [PSS, we show that, under the assumptions of the theorem above, the measures $P_{z} \nu$ and $\left.\mathcal{L}\right|_{P_{z}(K)}$ are equivalent for all $z \in S$. Here $\mathcal{L}$ is the Lebesgue measure on $\mathbb{R}$.

Finally, our results also apply to self-similar sets. We recall the following definition due to Furstenberg Fur. A subset $A \subset \mathbb{C}$ is said to be dimension conserving (DC), with respect to the projection $P_{z}$, if for some $\delta \geq 0$

$$
\delta+\operatorname{dim}_{H}\left\{x \in \mathbb{R}: \operatorname{dim}_{H}\left(A \cap P_{z}^{-1}\{x\}\right) \geq \delta\right\} \geq \operatorname{dim}_{H} A,
$$

where the dimension of the empty set is defined to be $-\infty$. From our results on measures we shall obtain the following corollary. A sharper version of it is given in Section 3 Note its close connection with the priorly mentioned result from [FJ2].

Corollary. Let $\mathcal{E} \subset \mathbb{D}$ be the 0 -dimensional set from the theorem above. Let $\lambda \in$ $\mathbb{D} \backslash \mathcal{E}$ be with $\arg \lambda \notin \pi \mathbb{Q}$, let $K$ be a self-similar set with the $S S C$ corresponding to an IFS as in (1.1), and assume that $s=\operatorname{dim}_{H} K>1$. Then there exists $c>0$ such that for every $z \in S$,

$$
\mathcal{L}\left\{x \in \mathbb{R}: \operatorname{dim}_{H}\left(K \cap P_{z}^{-1}\{x\}\right)=s-1\right\}>c .
$$

In particular, $K$ is $D C$ with respect to $P_{z}$ for all $z \in S$.

The rest of this paper is organized as follows. In Section 2 we give the necessary definitions. In Section 3 we state our results. In Section 4 we show that the projections $P_{z} \nu$ all belong to the appropriate function space, and establish the continuity of the map $z \rightarrow P_{z} \nu$. In Section 5 we prove the result regarding the slices of measures. In Section 6 we establish our results for self-similar sets. Finally, in Section 7 we prove the statement regarding the measure class of the projections.

Acknowledgment. I would like to thank P. Shmerkin for suggesting to consider a general $q>1$ in Theorem 3.1 instead of just $q=2$. I would also like to thank M. Hochman for helpful discussions.

\section{Preliminaries}

2.1. Self-similar sets and measures. Write $\mathbb{D}=\{z \in \mathbb{C}:|z|<1\}$ and $S=$ $\{z \in \mathbb{C}:|z|=1\}$. Given a metric space $X$, which will always be $\mathbb{C}$ or $\mathbb{R}$, denote by $\mathcal{M}(X)$ the collection of all compactly supported Borel probability measures on $X$. For a finite index set $\Lambda$ write

$$
\mathbb{P}_{\Lambda}=\left\{\left(p_{i}\right)_{i \in \Lambda} \in(0,1)^{\Lambda}: \sum_{i \in \Lambda} p_{i}=1\right\} .
$$

Given $p \in \mathbb{P}_{\Lambda},\left(a_{i}\right)_{i \in \Lambda}=\mathbf{a} \in \mathbb{C}^{\Lambda}$, and $\lambda \in \mathbb{D}$, let $\nu_{\lambda, \mathbf{a}}^{p} \in \mathcal{M}(\mathbb{C})$ be the self-similar measure corresponding to the IFS

$$
\mathcal{F}_{\lambda, \mathbf{a}}=\left\{f_{\lambda, \mathbf{a}}^{i}(z)=\lambda z+a_{i}\right\}_{i \in \Lambda}
$$

and $p$, i.e. $\nu_{\lambda, \mathbf{a}}^{p}$ is the unique member of $\mathcal{M}(\mathbb{C})$ such that

$$
\nu_{\lambda, \mathbf{a}}^{p}=\sum_{i \in \Lambda} p_{i} \cdot f_{\lambda, \mathbf{a}}^{i} \nu_{\lambda, \mathbf{a}}^{p} .
$$


Denote by $K_{\lambda, \mathbf{a}} \subset \mathbb{C}$ the attractor of $\mathcal{F}_{\lambda, \mathbf{a}}$, i.e. $K_{\lambda, \mathbf{a}}$ is the unique nonempty compact subset of $\mathbb{C}$ with

$$
K_{\lambda, \mathbf{a}}=\cup_{i \in \Lambda} f_{\lambda, \mathbf{a}}^{i}\left(K_{\lambda, \mathbf{a}}\right) .
$$

Since $p>0$ it holds that $K_{\lambda, \mathbf{a}}=\operatorname{supp}\left(\nu_{\lambda, \mathbf{a}}^{p}\right)$. We say that the strong separation condition (SSC) holds if the union in (2.2) is disjoint.

2.2. Projections and disintegrations. For $z, w \in \mathbb{C}$ write $\langle z, w\rangle=\operatorname{Re}(z \cdot \bar{w})$, then $\langle\cdot, \cdot\rangle$ is the standard inner product on $\mathbb{C}$ when it is identified with $\mathbb{R}^{2}$. For $z \in S$ let $P_{z}: \mathbb{C} \rightarrow \mathbb{R}$ be with $P_{z} w=\langle z, w\rangle$ for $w \in \mathbb{C}$. Note that given $\mu \in \mathcal{M}(\mathbb{C})$ the measure $P_{z} \mu$ is, up to affine equivalence, the pushforward of $\mu$ by the orthogonal projection onto the line $z \cdot \mathbb{R}$.

Denote by $\mathcal{B}$ the Borel $\sigma$-algebra of $\mathbb{R}$ or $\mathbb{C}$. For $\mu \in \mathcal{M}(\mathbb{C})$ and $z \in S$ let $\left\{\mu_{z, w}\right\}_{w \in \mathbb{C}} \subset \mathcal{M}(\mathbb{C})$ be the disintegration of $\mu$ with respect to $P_{z}^{-1}(\mathcal{B})$, as defined in Theorem 5.14 in [EW]. This means that $\mu_{z, w}$ is supported on $P_{z}^{-1}\left(P_{z} w\right)$ for $\mu$-a.e. $w \in \mathbb{C}$, and that for each bounded $\mathcal{B}$-measurable $f: \mathbb{C} \rightarrow \mathbb{R}$

$$
\int f d \mu_{z, w}=E_{\mu}\left(f \mid P_{z}^{-1}(\mathcal{B})\right)(w) \text { for } \mu \text {-a.e. } w \in \mathbb{C} .
$$

In the last equality, the right hand side is the conditional expectation of $f$ given $P_{z}^{-1}(\mathcal{B})$ with respect to $\mu$.

2.3. Dimension of measures. Let $X$ be $\mathbb{C}$ or $\mathbb{R}$. For $E \subset X$ denote by $\operatorname{dim}_{H} E$ the Hausdorff dimension of $E$. Given $\mu \in \mathcal{M}(X)$ write $\operatorname{dim}_{H} \mu$ for the Hausdorff dimension of $\mu$, which is defined by

$$
\operatorname{dim}_{H} \mu=\inf \left\{\operatorname{dim}_{H} E: E \subset X \text { is a Borel set with } \mu(E)>0\right\} .
$$

For $x \in X$ and $\delta>0$ let $B(x, \delta)$ be the closed ball in $X$ with centre $x$ and radius $\delta$. It is said that $\mu$ has exact dimension $s \geq 0$ if

$$
\lim _{\delta \downarrow 0} \frac{\log \mu(B(x, \delta))}{\log \delta}=s \text { for } \mu \text {-a.e. } x \in X .
$$

It is well known that in this case $s=\operatorname{dim}_{H} \mu$ (see [Fal2, Chapter 10]).

For $1<q<\infty$ denote by $D_{q}(\mu)$ the lower $L^{q}$ dimension of $\mu$, which is defined by

$$
D_{q}(\mu)=\liminf _{\delta \downarrow 0} \frac{\log \int(\mu(B(x, \delta)))^{q-1} d \mu(x)}{(q-1) \log \delta} .
$$

It always holds that $\operatorname{dim}_{H} \mu \geq D_{q}(\mu)$ and $D_{q^{\prime}}(\mu) \geq D_{q}(\mu)$ for all $1<q^{\prime}<q$. A proof of these standard facts can be found in [FLR]. The number $D_{2}(\mu)$ is called the lower correlation dimension of $\mu$.

For $p \in \mathbb{P}_{\Lambda}$ write $\|p\|_{q}^{q}=\sum_{i \in \Lambda} p_{i}^{q}$. It is not hard to verify that for $p \in \mathbb{P}_{\Lambda}$, $\mathbf{a} \in \mathbb{C}^{\Lambda}$, and $\lambda \in \mathbb{D}$, such that the SSC is satisfied,

$$
\begin{aligned}
D_{q}\left(\nu_{\lambda, \mathbf{a}}^{p}\right) & =\frac{\log \|p\|_{q}^{q}}{(q-1) \log |\lambda|}, \\
\operatorname{dim}_{H} \nu_{\lambda, \mathbf{a}}^{p} & =\frac{\sum_{i \in \Lambda} p_{i} \log p_{i}}{\log |\lambda|},
\end{aligned}
$$

and

$$
\operatorname{dim}_{H} K_{\lambda, \mathbf{a}}=\frac{\log |\Lambda|}{-\log |\lambda|}
$$


2.4. Function spaces. Let $\mathcal{L}$ be the Lebesgue measure on $\mathbb{R}$. For $1 \leq p \leq \infty$ denote by $\|\cdot\|_{p}$ the $L^{p}$-norm on $L^{p}(\mathbb{R})$. Given $g \in L^{2}(\mathbb{R})$ write $\widehat{g}$ for the Fourier transform of $g$, which is again a member of $L^{2}(\mathbb{R})$.

For $\gamma \in[0, \infty)$ denote by $H_{\gamma}(\mathbb{R})$ the $2, \gamma$-Sobolev space of $\mathbb{R}$, i.e.

$$
H_{\gamma}(\mathbb{R})=\left\{g \in L^{2}(\mathbb{R}): \int|\widehat{g}(\xi)|^{2}\left(1+|\xi|^{2}\right)^{\gamma} d \xi<\infty\right\} .
$$

For $g \in H_{\gamma}(\mathbb{R})$ write,

$$
\|g\|_{(\gamma)}=\left(\int|\widehat{g}(\xi)|^{2}\left(1+|\xi|^{2}\right)^{\gamma} d \xi\right)^{1 / 2} .
$$

Note that $\left(H_{\gamma}(\mathbb{R}),\|\cdot\|_{(\gamma)}\right)$ is a Hilbert space, $H_{\gamma}(\mathbb{R}) \subset H_{\gamma^{\prime}}(\mathbb{R})$ and $\|g\|_{\left(\gamma^{\prime}\right)} \leq\|g\|_{(\gamma)}$ for each $0 \leq \gamma^{\prime} \leq \gamma$, and $H_{0}(\mathbb{R})=L^{2}(\mathbb{R})$.

Given $\mu \in \mathcal{M}(\mathbb{R})$ and a Banach space $B$ of functions on $\mathbb{R}$, we write $\mu \in B$ when $\mu$ is absolutely continuous and its density belongs to $B$.

2.5. The exceptional set of parameters. All of our results will be valid for selfsimilar measures $\nu_{\lambda, \mathbf{a}}^{p}$ and sets $K_{\lambda, \mathbf{a}}$ for which $\lambda \in \mathbb{D}$ lies outside a 0-dimensional set $\mathcal{E}$. We shall now define this exceptional set of parameters.

For $X$ which is $\mathbb{R}$ or $\mathbb{C}$ and $\mu \in \mathcal{M}(X)$ write $\widehat{\mu}$ for the Fourier transform of $\mu$, i.e. if $X=\mathbb{R}$

and if $X=\mathbb{C}$

$$
\widehat{\mu}(\xi)=\int e^{i \xi x} d \mu(x) \text { for } \xi \in \mathbb{R},
$$

$$
\widehat{\mu}(\xi)=\int e^{i\langle\xi, w\rangle} d \mu(w) \text { for } \xi \in \mathbb{C} .
$$

By Theorem D in [SS2] there exists a set $\mathcal{E}^{\prime} \subset \mathbb{D}$, with $\operatorname{dim}_{H} \mathcal{E}^{\prime}=0$, such that the following holds. Let $\lambda \in \mathbb{D} \backslash\left(\mathcal{E}^{\prime} \cup \mathbb{R}\right), \Lambda$ a finite nonempty set, $\left(a_{i}\right)_{i \in \Lambda}=\mathbf{a} \in \mathbb{C}^{\Lambda}$ with not all of the $a_{i}$ equal, and $p \in \mathbb{P}_{\Lambda}$. Then there are $C, \gamma>0$ such that

$$
\left|\widehat{\nu_{\lambda, \mathbf{a}}^{p}}(\xi)\right| \leq C|\xi|^{-\gamma} \text { for all } \xi \in \mathbb{C} .
$$

Write

then $\operatorname{dim}_{H} \mathcal{E}=0$.

$$
\mathcal{E}=\cup_{k \geq 1}\left\{\lambda \in \mathbb{D}: \lambda^{k} \in \mathcal{E}^{\prime}\right\}
$$

\section{Statement of Results}

The following theorem is our main result. Parts (1) and (2) are proven in Section 4. part (3) is proven in Section [5, and part (4) is proven in Section 7.

Theorem 3.1. Let $\lambda \in \mathbb{D} \backslash \mathcal{E}$ be with $\arg \lambda \notin \pi \mathbb{Q}, \Lambda$ a finite nonempty set, $\mathbf{a} \in \mathbb{C}^{\Lambda}$ such that $\mathcal{F}_{\lambda, \mathbf{a}}$ satisfies the $S S C$, and $p \in \mathbb{P}_{\Lambda}$ with $D_{q}\left(\nu_{\lambda, \mathbf{a}}^{p}\right)>1$ for some $1<q<\infty$. Set $\nu=\nu_{\lambda, \mathbf{a}}^{p}$, then

(1) $P_{z} \nu \in L^{q}(\mathbb{R})$ for all $z \in S$;

(2) $z \rightarrow P_{z} \nu$ is continuous as a map from $S$ to $L^{q}(\mathbb{R})$ with respect to the weak topology of $L^{q}(\mathbb{R})$

(3) for every $z \in S$ the measure $\nu_{z, w}$ has exact dimension $\operatorname{dim}_{H} \nu-1$ for $\nu$-a.e. $w \in \mathbb{C}$

(4) for every $z \in S$ the measures $P_{z} \nu$ and $\mathcal{L}_{P_{z}\left(K_{\lambda, \mathbf{a}}\right)}$ are equivalent. 
By differentiation it follows that for every probability vector $p \in \mathbb{P}_{\Lambda}$,

$$
\lim _{q \downarrow 1} \frac{\log \|p\|_{q}^{q}}{(q-1)}=\sum_{i \in \Lambda} p_{i} \log p_{i} .
$$

Hence by (2.3) and (2.4) we get the following direct corollary of Theorem 3.1

Corollary 3.2. Let $\lambda \in \mathbb{D} \backslash \mathcal{E}$ be with $\arg \lambda \notin \pi \mathbb{Q}, \Lambda$ a finite nonempty set, a $\in \mathbb{C}^{\Lambda}$ such that $\mathcal{F}_{\lambda, \mathbf{a}}$ satisfies the $S S C$, and $p \in \mathbb{P}_{\Lambda}$ with $\operatorname{dim}_{H} \nu_{\lambda, \mathbf{a}}^{p}>1$. Then there exists $1<q<\infty$ such that parts (11) to (4) in Theorem 3.1 are valid for $\nu=\nu_{\lambda, \mathbf{a}}^{p}$.

Remark 3.3. The following observation might be of interest. Let $\mathcal{F}_{\lambda, \mathbf{a}}$ be an IFS as in Theorem 3.1 and $p_{1}, p_{2} \in \mathbb{P}_{\Lambda}$ with $p_{1} \neq p_{2}$. Set $\nu_{1}=\nu_{\lambda, \mathbf{a}}^{p_{1}}$ and $\nu_{2}=\nu_{\lambda, \mathbf{a}}^{p_{2}}$, and assume

$$
\operatorname{dim}_{H} \nu_{1}, \operatorname{dim}_{H} \nu_{2}>1 \text {. }
$$

It holds that $\nu_{1}$ and $\nu_{2}$ are singular. This follows from the fact that they are both ergodic with respect to an appropriate map from $K_{\lambda, \mathbf{a}}$ onto itself (see Section 5) and since $\nu_{1} \neq \nu_{2}$. On the other hand, by part (4) of Theorem 3.1. $P_{z} \nu_{1}$ is equivalent to $P_{z} \nu_{2}$ for each $z \in S$.

From Theorem 3.1 we obtain the following result for self-similar sets. Its proof is given in Section 6. For $s \geq 0$ denote by $\mathcal{H}^{s}$ and $\mathcal{P}^{s}$ the $s$-dimensional Hausdorff and packing measures respectively. It is well known that $0<\mathcal{H}^{s}(K)<\infty$ whenever $K$ is a self-similar set with the SSC and dimension $s$. Given $z \in S$ and $x \in \mathbb{R}$ we write $K_{z, x}=K \cap P_{z}^{-1}\{x\}$.

Corollary 3.4. Let $\lambda \in \mathbb{D} \backslash \mathcal{E}$ be with $\arg \lambda \notin \pi \mathbb{Q}, \Lambda$ a finite nonempty set, and $\mathbf{a} \in \mathbb{C}^{\Lambda}$ such that $\mathcal{F}_{\lambda, \mathbf{a}}$ satisfies the SSC. Set $K=K_{\lambda, \mathbf{a}}$ and $s=\operatorname{dim}_{H} K$, and assume $s>1$. Then,

(1) for each $z \in S$

$$
\operatorname{dim}_{H} K_{z, P_{z} w}=s-1 \text { and } \mathcal{P}^{s-1}\left(K_{z, P_{z} w}\right)>0 \text { for } \mathcal{H}^{s} \text {-a.e. } w \in K
$$

(2) there exists $c>0$ such that for every $z \in S$,

$$
\mathcal{L}\left\{x \in \mathbb{R}: \operatorname{dim}_{H} K_{z, x}=s-1 \text { and } \mathcal{P}^{s-1}\left(K_{z, x}\right)>0\right\}>c .
$$

Remark 3.5. In [Rap1, Theorem 2.4] the author has shown that, under the assumptions of the last corollary, for Lebesgue almost every $z \in S$

$$
\mathcal{P}^{s-1}\left(K_{z, P_{z} w}\right)>0 \text { for } \mathcal{H}^{s} \text {-a.e. } w \in K .
$$

In Corollary 3.4 this is established for all $z \in S$.

Remark 3.6. If the last corollary would remain true with $\mathcal{H}^{s-1}$ instead of $\mathcal{P}^{s-1}$, then by [Rap1, Corollary 2.3] it would follow that $P_{z}\left(\left.\mathcal{H}^{s}\right|_{K_{\lambda, \mathbf{a}}}\right) \in L^{\infty}(\mathbb{R})$ for all $z \in S$ and that

$$
\sup _{z \in S}\left\|P_{z}\left(\left.\mathcal{H}^{s}\right|_{K_{\lambda, \mathbf{a}}}\right)\right\|_{\infty}<\infty .
$$

Unfortunately, currently we are unable to prove this stronger statement.

If in Theorem 3.1 we assume that the correlation dimension $D_{2}\left(\nu_{\lambda, \mathbf{a}}^{p}\right)$ exceeds 1 then we get the following result. It guarantees the continuity of the map $z \rightarrow P_{z} \nu$ with respect to the norm topology, which is of course much stronger than continuity with respect to the weak topology. The proof is given in Section 4 
Theorem 3.7. Let $\lambda \in \mathbb{D} \backslash \mathcal{E}$ be with $\arg \lambda \notin \pi \mathbb{Q}, \Lambda$ a finite nonempty set, $\mathbf{a} \in \mathbb{C}^{\Lambda}$ such that $\mathcal{F}_{\lambda, \mathbf{a}}$ satisfies the $S S C$, and $p \in \mathbb{P}_{\Lambda}$ with $D_{2}\left(\nu_{\lambda, \mathbf{a}}^{p}\right)>1$. Set $\nu=\nu_{\lambda, \mathbf{a}}^{p}$, then there exists $\gamma>0$ such that

(1) $P_{z} \nu \in H_{\gamma}(\mathbb{R})$ for all $z \in S$;

(2) $z \rightarrow P_{z} \nu$ is continuous as a map from $S$ to $\left(H_{\gamma}(\mathbb{R}),\|\cdot\|_{(\gamma)}\right)$.

Remark 3.8. Note that part (2) of the last theorem implies that $z \rightarrow P_{z} \nu$ is continuous as a map from $S$ to $\left(L^{2}(\mathbb{R}),\|\cdot\|_{2}\right)$. This in turn implies the continuity of this map with respect to the total variation norm on $\mathcal{M}(\mathbb{R})$.

Let $\lambda \in \mathbb{D}$ and $\mathbf{a} \in \mathbb{C}^{\Lambda}$ be such that $\mathcal{F}_{\lambda, \mathbf{a}}$ satisfies the SSC, and write $s=$ $\operatorname{dim}_{H} K_{\lambda, \mathbf{a}}$ and $p^{\prime}=\left(\frac{1}{|\Lambda|}, \ldots, \frac{1}{|\Lambda|}\right)$. It is not hard to see that there exists $0<c<\infty$ so that

$$
\left.\mathcal{H}^{s}\right|_{K_{\lambda, \mathbf{a}}}=c \cdot \nu_{\lambda, \mathbf{a}}^{p^{\prime}} .
$$

Also, for each $1<q<\infty$ it follows by (2.3) and (2.5) that

$$
D_{q}\left(\nu_{\lambda, \mathbf{a}}^{p^{\prime}}\right)=\frac{\log |\Lambda|}{-\log |\lambda|}=\operatorname{dim}_{H} K_{\lambda, \mathbf{a}} .
$$

From these facts we get the following direct corollary of Theorems 3.1 and 3.7. It shows that when $s>1$ the projections of $\left.\mathcal{H}^{s}\right|_{K_{\lambda, \mathbf{a}}}$ are very regular.

Corollary 3.9. Let $\lambda \in \mathbb{D} \backslash \mathcal{E}$ be with $\arg \lambda \notin \pi \mathbb{Q}, \Lambda$ a finite nonempty set, and $\mathbf{a} \in \mathbb{C}^{\Lambda}$ such that $\mathcal{F}_{\lambda, \mathbf{a}}$ satisfies the SSC. Set $s=\operatorname{dim}_{H} K_{\lambda, \mathbf{a}}$ and assume $s>1$. Then for $\nu=\left.\mathcal{H}^{s}\right|_{K_{\lambda, \mathbf{a}}}$ and every $1<q<\infty$, parts (1) to (4) of Theorem 3.1 are valid. Additionally, parts (11) and (2) of Theorem 3.7 are valid for some $\gamma>0$.

\section{Proof of Parts (11) and (21) of Theorems 3.1 and 3.7}

The following two results will be needed. Let $X$ be $\mathbb{C}$ or $\mathbb{R}$ and for $\mu, \nu \in \mathcal{M}(X)$ write $\mu * \nu$ for their convolution.

Theorem 4.1 ([SS1, Theorem 4.4). Let $1<q<\infty, \mu \in \mathcal{M}(\mathbb{R})$ with $D_{q}(\mu)=1$, and $\nu \in \mathcal{M}(\mathbb{R})$ such that there exist $C, \gamma>0$ with $|\widehat{\nu}(\xi)| \leq C|\xi|^{-\gamma}$ for all $\xi \in \mathbb{R}$. Then $\mu * \nu \in L^{q}(\mathbb{R})$.

Lemma 4.2 ([Sh1], Lemma 2.1). Let $\mu \in \mathcal{M}(\mathbb{R})$ with $D_{2}(\mu)=1$ and $\nu \in \mathcal{M}(\mathbb{R})$ such that there exist $C, \gamma>0$ with $|\widehat{\nu}(\xi)| \leq C|\xi|^{-\gamma}$ for all $\xi \in \mathbb{R}$. Then $\mu * \nu \in$ $H_{\gamma / 4}(\mathbb{R})$.

In the next proposition we prove the first part of Theorems 3.1 and 3.7. The proof follows an idea introduced in [Sh1]. It also relies on a result from [Sh2] regarding the $L^{q}$ dimensions of projections of planar self-similar measures.

Proposition 4.3. Let $\lambda \in \mathbb{D} \backslash \mathcal{E}$ be with $\arg \lambda \notin \pi \mathbb{Q}, \Lambda$ a finite nonempty set, $\left(a_{i}\right)_{i \in \Lambda}=\mathbf{a} \in \mathbb{C}^{\Lambda}$ such that $\mathcal{F}_{\lambda, \mathbf{a}}$ satisfies the $S S C$, and $p \in \mathbb{P}_{\Lambda}$. Set $\nu=\nu_{\lambda, \mathbf{a}}^{p}$, then

(1) if $1<q<\infty$ is such that $D_{q}(\nu)>1$ then $P_{z} \nu \in L^{q}(\mathbb{R})$ for all $z \in S$;

(2) if $D_{2}(\nu)>1$ then there exists $\gamma>0$ such that $P_{z} \nu \in H_{\gamma}(\mathbb{R})$ for all $z \in S$.

Proof. Without loss of generality we can assume that $a_{i}=0$ for some $i \in \Lambda$. Otherwise we can arrange this by replacing $\mathcal{F}_{\lambda, \mathbf{a}}=\left\{f_{\lambda, \mathbf{a}}^{i}\right\}_{i \in \Lambda}$ with

$$
\left\{h \circ f_{\lambda, \mathbf{a}}^{i} \circ h^{-1}\right\}_{i \in \Lambda}
$$

and $\nu$ with $h \nu$, where $h: \mathbb{C} \rightarrow \mathbb{C}$ is of the form $h(w)=w+\beta$ for some $\beta \in \mathbb{C}$. 
Assume that $1<q<\infty$ satisfies $D_{q}(\nu)>1$. Let $k \geq 2$ be with $\left(1-\frac{1}{k}\right) D_{q}(\nu)>1$. For $\left(i_{1}, \ldots, i_{k-1}\right)=\boldsymbol{i} \in \Lambda^{k-1}$ write $p_{\boldsymbol{i}}=p_{i_{1}} \cdot \ldots \cdot p_{i_{k-1}}$ and

$$
g_{i}(w)=\lambda^{k} w+\sum_{j=1}^{k-1} a_{i_{j}} \lambda^{j} \text { for } w \in \mathbb{C} .
$$

Since $\mathcal{F}_{\lambda, \mathbf{a}}$ satisfies the SSC and $a_{i}=0$ for some $i \in \Lambda$, it is easy to see that $\left\{g_{\boldsymbol{i}}\right\}_{\boldsymbol{i} \in \Lambda^{k-1}}$ also satisfies the SSC. Let $\mu \in \mathcal{M}(\mathbb{C})$ be the self-similar measure corresponding to $\left\{g_{\boldsymbol{i}}\right\}_{\boldsymbol{i} \in \Lambda^{k-1}}$ and $\left\{p_{\boldsymbol{i}}\right\}_{\boldsymbol{i} \in \Lambda^{k-1}}$, i.e.

$$
\mu=\sum_{i \in \Lambda^{k-1}} p_{i} \cdot g_{i} \mu
$$

By (2.3),

$$
\begin{aligned}
D_{q}(\mu) & =\frac{\log \left\|\left(p_{i}\right)_{\boldsymbol{i} \in \Lambda^{k-1}}\right\|_{q}^{q}}{(q-1) \log \left|\lambda^{k}\right|} \\
& =\frac{(k-1) \log \|p\|_{q}^{q}}{k(q-1) \log |\lambda|}=\left(1-\frac{1}{k}\right) D_{q}(\nu)>1 .
\end{aligned}
$$

Hence by Theorem 8.2 in [Sh2],

$$
D_{q}\left(P_{z} \mu\right)=1 \text { for all } z \in S .
$$

Recall the definition of the sets $\mathcal{E}$ and $\mathcal{E}^{\prime}$ from Section 2.5. Since $\lambda \notin \mathcal{E}$ and $\arg \lambda \notin \pi \mathbb{Q}$ we have $\lambda^{k} \notin \mathcal{E}^{\prime} \cup \mathbb{R}$. Hence there exist $C, \gamma>0$ such that,

$$
\left|\widehat{\nu_{\lambda^{k}, \mathbf{a}}^{p}}(\xi)\right| \leq C|\xi|^{-\gamma} \text { for all } \xi \in \mathbb{C} .
$$

Let $z \in S$, then a direct computation shows that

$$
\widehat{P_{z} \nu_{\lambda^{k}, \mathbf{a}}^{p}}(t)=\widehat{\nu_{\lambda^{k}, \mathbf{a}}^{p}}(t z) \text { for all } t \in \mathbb{R},
$$

and so

$$
\left|\widehat{P_{z} \nu_{\lambda^{k}, \mathbf{a}}^{p}}(t)\right| \leq C|t|^{-\gamma} \text { for all } t \in \mathbb{R} .
$$

Note that $\nu=\mu * \nu_{\lambda^{k}, \mathbf{a}}^{p}$, hence $P_{z} \nu=P_{z} \mu * P_{z} \nu_{\lambda^{k}, \mathbf{a}}^{p}$. From this, (4.1), (4.2), and Theorem 4.1. it now follows $P_{z} \nu \in L^{q}(\mathbb{R})$, which completes the proof of the first part.

The proof of the second part is similar, except that at the end of the proof one needs to use Lemma 4.2 instead of Theorem 4.1 .

We now turn to the proof of the second part of Theorems 3.1 and 3.7. Throughout this section the pair $\left(B,\|\cdot\|_{B}\right)$ will denote $\left(L^{q}(\mathbb{R}),\|\cdot\|_{q}\right)$ for some $1<q<\infty$ or $\left(H_{\gamma}(\mathbb{R}),\|\cdot\|_{(\gamma)}\right)$ for some $0 \leq \gamma<\infty$. We write $C_{c}(\mathbb{R})$ for the collection of all compactly supported continuous functions on $\mathbb{R}$.

Lemma 4.4. Let $\nu \in \mathcal{M}(\mathbb{C})$ and $C>0$ be given, and set

$$
F=\left\{z \in S: P_{z} \nu \in B \text { and }\left\|\frac{d P_{z} \nu}{d \mathcal{L}}\right\|_{B} \leq C\right\} .
$$

Then $F$ is a closed subset of $S$. 
Proof. For every $z \in F$ there exists $g_{z} \in B$ with $d P_{z} \nu=g_{z} d \mathcal{L}$ and $\left\|g_{z}\right\|_{B} \leq C$. Let $z_{1}, z_{2}, \ldots \in F$ and $z \in S$ be with $z_{k} \stackrel{k}{\rightarrow} z$ as $k \rightarrow \infty$. Note that $\left\{g_{z_{k}}\right\}_{k \geq 1}$ is a bounded sequence in the reflexive Banach space $B$. Hence, by moving to a subsequence without changing notation, we may assume that there exists $g \in B$ such that

$$
g_{z_{k}} \stackrel{k}{\rightarrow} g \text { weakly in } B \text { as } k \rightarrow \infty .
$$

Additionally, by (4.3)

$$
\|g\|_{B} \leq \liminf _{k}\left\|g_{z_{k}}\right\|_{B} \leq C .
$$

Given $h \in C_{c}(\mathbb{R})$ we get from (4.3),

$$
\int h(x) g(x) d x=\lim _{k} \int h(x) g_{z_{k}}(x) d x=\lim _{k} \int h\left(P_{z_{k}} w\right) d \nu(w) .
$$

Hence, by bounded convergence and since $P_{z_{k}} w \stackrel{k}{\rightarrow} P_{z} w$ for all $w \in \mathbb{C}$,

$$
\int h(x) g(x) d x=\int h\left(P_{z} w\right) d \nu(w)=\int h(x) d P_{z} \nu(x) .
$$

This shows $d P_{z} \nu=g d \mathcal{L}$, which implies that $z \in F$ and completes the proof of the lemma.

For $b \in \mathbb{R}$ and $c>0$ let

$$
\tau_{b}(x)=x-b \text { and } M_{c}(x)=c x \text { for } x \in \mathbb{R} .
$$

In what follows $\Lambda$ stands for some finite nonempty index set. Recall the definition of the IFS $\mathcal{F}_{\lambda, \mathbf{a}}$ from (2.1), which consists of maps $f_{i}=f_{\lambda, \mathbf{a}}^{i}$ for $i \in \Lambda$. Given a word $i_{1} \ldots i_{k}=\boldsymbol{i} \in \Lambda^{*}$ write $f_{\boldsymbol{i}}=f_{i_{1}} \circ \ldots \circ f_{i_{k}}$, and for $\left(p_{i}\right)_{i \in \Lambda}=p \in \mathbb{P}_{\Lambda}$ set $p_{\boldsymbol{i}}=p_{i_{1}} \cdot \ldots \cdot p_{i_{k}}$.

Lemma 4.5. Let $\alpha \in S, 0<r<1, \mathbf{a} \in \mathbb{C}^{\Lambda}$, and $p \in \mathbb{P}_{\Lambda}$. Write $\lambda=r \alpha, \nu=\nu_{\lambda, \mathbf{a}}^{p}$, and $f_{i}=f_{\lambda, \mathbf{a}}^{i}$ for every $i \in \Lambda$. Assume that $P_{z} \nu$ is absolutely continuous for each $z \in S$ and write $g_{z}=\frac{d P_{z} \nu}{d \mathcal{L}}$. Let $z \in S$ and $k \geq 1$, and for each $\boldsymbol{i} \in \Lambda^{k}$ set $b_{\boldsymbol{i}}=P_{z} f_{\boldsymbol{i}}(0)$. Then for $\mathcal{L}$-a.e. $x \in \mathbb{R}$,

$$
g_{z}(x)=\sum_{i \in \Lambda^{k}} p_{i} r^{-k} \cdot g_{\alpha^{-k} z}\left(\tau_{r^{-k} b_{i}} M_{r^{-k}} x\right) .
$$

Proof. For $h \in C_{c}(\mathbb{R})$,

$$
\int h(x) g_{z}(x) d x=\int h\left(P_{z} w\right) d \nu(w)=\sum_{\boldsymbol{i} \in \Lambda^{k}} p_{\boldsymbol{i}} \int h\left(P_{z} w\right) d f_{\boldsymbol{i}} \nu(w) .
$$

For $i \in \Lambda^{k}$,

$$
\begin{aligned}
\int h\left(P_{z} f_{\boldsymbol{i}} w\right) d \nu(w)=\int h\left(b_{\boldsymbol{i}}+r^{k} P_{\alpha^{-k} z} w\right) d \nu(w) & \\
=\int h\left(b_{\boldsymbol{i}}+r^{k} x\right) d P_{\alpha^{-k} z} \nu(x) & =\int h\left(b_{\boldsymbol{i}}+r^{k} x\right) g_{\alpha^{-k} z}(x) d x \\
& =\int h(x) g_{\alpha^{-k} z}\left(r^{-k} x-r^{-k} b_{\boldsymbol{i}}\right) r^{-k} d x .
\end{aligned}
$$

By combining these equalities,

$$
\int h(x) g_{z}(x) d x=\int h(x) \sum_{i \in \Lambda^{k}} p_{i} r^{-k} \cdot g_{\alpha^{-k} z}\left(\tau_{r^{-k} b_{i}} M_{r^{-k}} x\right) d x .
$$


Now since this holds for every $h \in C_{c}(\mathbb{R})$ the lemma follows.

Lemma 4.6. There exists $\beta \in \mathbb{R}$ such that for every $g \in B, b \in \mathbb{R}$ and $c \geq 1$,

$$
\left\|g \circ \tau_{b} \circ M_{c}\right\|_{B} \leq c^{\beta} \cdot\|g\|_{B} .
$$

Proof. Let $g \in B, b \in \mathbb{R}$ and $c \geq 1$. If $B=L^{q}(\mathbb{R})$ for $1<q<\infty$, then

$$
\left\|g \circ \tau_{b} \circ M_{c}\right\|_{B}=c^{-1 / q}\|g\|_{B} .
$$

If $B=H_{\gamma}(\mathbb{R})$ for $0 \leq \gamma<\infty$, then

$$
\begin{aligned}
\left\|g \circ \tau_{b} \circ M_{c}\right\|_{B}^{2} & =\int\left|\left(g \circ \tau_{b} \circ M_{c}\right)^{\wedge}(\xi)\right|^{2}\left(1+|\xi|^{2}\right)^{\gamma} d \xi \\
& =\int\left|c^{-1} \cdot e^{i b \xi / c} \cdot \widehat{g}(\xi / c)\right|^{2}\left(1+|\xi|^{2}\right)^{\gamma} d \xi \\
& =c^{-1} \int|\widehat{g}(\xi)|^{2}\left(1+|c \xi|^{2}\right)^{\gamma} d \xi \leq c^{2 \gamma-1}\|g\|_{B}^{2},
\end{aligned}
$$

which proves the lemma.

The following key proposition will be used several times below. Recall that $\left(B,\|\cdot\|_{B}\right)$ denotes $\left(L^{q}(\mathbb{R}),\|\cdot\|_{q}\right)$ for some $1<q<\infty$ or $\left(H_{\gamma}(\mathbb{R}),\|\cdot\|_{(\gamma)}\right)$ for some $0 \leq \gamma<\infty$.

Proposition 4.7. Let $\lambda \in \mathbb{D}$ be with $\arg \lambda \notin \pi \mathbb{Q}, \mathbf{a} \in \mathbb{C}^{\Lambda}$, and $p \in \mathbb{P}_{\Lambda}$. Set $\nu=\nu_{\lambda, \mathbf{a}}^{p}$ and assume that $P_{z} \nu \in B$ for all $z \in S$. Then $\left\{P_{z} \nu\right\}_{z \in S}$ is a bounded subset of $\left(B,\|\cdot\|_{B}\right)$.

Proof. Set $f_{i}=f_{\lambda, \mathbf{a}}^{i}$ for every $i \in \Lambda$. By assumption, for every $z \in S$ there exists $g_{z} \in B$ with $d P_{z} \nu=g_{z} d \mathcal{L}$. For $n \geq 1$ let

$$
F_{n}=\left\{z \in S:\left\|g_{z}\right\|_{B} \leq n\right\},
$$

then $F_{n}$ is closed in $S$ by Lemma 4.4 .

From $S=\cup_{n \geq 1} F_{n}$ and Baire's theorem, it follows that there exist $n \geq 1$ and an open nonempty subset $V$ of $S$ with $V \subset F_{n}$. Let $\alpha \in S$ and $0<r<1$ be with $\lambda=r \alpha$. Since

$$
\arg \alpha=\arg \lambda \notin \pi \mathbb{Q},
$$

there exits $N \geq 1$ such that for each $z \in S$ there exists $1 \leq k \leq N$ with $\alpha^{-k} z \in V$. Fix $z \in S$ and let $1 \leq k \leq N$ be with $\alpha^{-k} z \in V$.

For $\boldsymbol{i} \in \Lambda^{k}$ write $b_{\boldsymbol{i}}=P_{z} f_{\boldsymbol{i}}(0)$. By Lemma 4.5 it follows that for $\mathcal{L}$-a.e. $x \in \mathbb{R}$,

$$
g_{z}(x)=\sum_{i \in \Lambda^{k}} p_{i} r^{-k} \cdot g_{\alpha^{-k} z}\left(\tau_{r^{-k} b_{i}} M_{r^{-k}} x\right) .
$$

Hence by Lemma 4.6 and since $\alpha^{-k} z \in V \subset F_{n}$,

$$
\begin{aligned}
\left\|g_{z}\right\|_{B} & \leq \sum_{i \in \Lambda^{k}} p_{i} r^{-k} \cdot\left\|g_{\alpha^{-k} z} \circ \tau_{r^{-k} b_{\boldsymbol{i}}} \circ M_{r^{-k}}\right\|_{B} \\
& \leq r^{-k(1+\beta)} \cdot\left\|g_{\alpha^{-k} z}\right\|_{B} \leq n \cdot \max \left\{r^{-N(1+\beta)}, 1\right\},
\end{aligned}
$$

which completes the proof of the proposition.

Part (2) of Theorems 3.1 and 3.7 will follow directly from the following two lemmas and Propositions 4.3 and 4.7. 
Lemma 4.8. Let $\nu \in \mathcal{M}(\mathbb{C}), 1<q<\infty$ and $C>0$. Assume that $P_{z} \nu \in L^{q}(\mathbb{R})$, with $\left\|P_{z} \nu\right\|_{q} \leq C$, for each $z \in S$. Then $z \rightarrow P_{z} \nu$ is continuous as a map from $S$ to $L^{q}(\mathbb{R})$, with respect to the weak topology of $L^{q}(\mathbb{R})$.

Proof. By assumption for every $z \in S$ there exists $g_{z} \in L^{q}(\mathbb{R})$ with $d P_{z} \nu=g_{z} d \mathcal{L}$. Moreover,

$$
\left\|g_{z}\right\|_{q} \leq C \text { for all } z \in S .
$$

Let $z \in S$ and $\left\{z_{0, k}\right\}_{k \geq 1} \subset S$ be with $z_{0, k} \stackrel{k}{\rightarrow} z$. It suffices to show that

$$
g_{z_{0, k}} \stackrel{k}{\rightarrow} g_{z} \text { weakly in } L^{q}(\mathbb{R}) \text { as } k \rightarrow \infty .
$$

Let $\left\{z_{1, k}\right\}_{k \geq 1}$ be a subsequence of $\left\{z_{0, k}\right\}_{k \geq 1}$. From (4.4) and since $L^{q}(\mathbb{R})$ is reflexive, it follows that there exist a subsequence $\left\{z_{2, k}\right\}_{k \geq 1}$ of $\left\{z_{1, k}\right\}_{k \geq 1}$ and $g \in$ $L^{q}(\mathbb{R})$ such that $g_{z_{2, k}} \stackrel{k}{\rightarrow} g$ weakly in $L^{q}(\mathbb{R})$ as $k \rightarrow \infty$.

Since $z_{2, k} \stackrel{k}{\rightarrow} z$, it holds for each $h \in C_{c}(\mathbb{R})$ that

$$
\begin{aligned}
\int h(x) g(x) d x & =\lim _{k} \int h(x) g_{z_{2, k}}(x) d x \\
& =\lim _{k} \int h\left(P_{z_{2, k}} w\right) d \nu(w)=\int h\left(P_{z} w\right) d \nu(w)=\int h(x) g_{z}(x) d x .
\end{aligned}
$$

Hence $g=g_{z}$, and so $g_{z_{2, k}} \stackrel{k}{\rightarrow} g_{z}$ weakly as $k \rightarrow \infty$. Since $\left\{z_{1, k}\right\}_{k \geq 1}$ was an arbitrary subsequence of $\left\{z_{0, k}\right\}_{k \geq 1}$ this gives (4.5) and completes the proof of the lemma.

Lemma 4.9. Let $\nu \in \mathcal{M}(\mathbb{C})$ and $\gamma, C>0$. Assume that $P_{z} \nu \in H_{\gamma}(\mathbb{R})$, with $\left\|P_{z} \nu\right\|_{(\gamma)} \leq C$, for all $z \in S$. Let $\gamma^{\prime} \in(0, \gamma)$, then $z \rightarrow P_{z} \nu$ is continuous as a map from $S$ to $\left(H_{\gamma^{\prime}}(\mathbb{R}),\|\cdot\|_{\left(\gamma^{\prime}\right)}\right)$.

Proof. By assumption for every $z \in S$ there exists $g_{z} \in H_{\gamma}(\mathbb{R})$ with $d P_{z} \nu=g_{z} d \mathcal{L}$. Moreover,

$$
\left\|g_{z}\right\|_{(\gamma)} \leq C \text { for all } z \in S .
$$

Let $z \in S$ and $\left\{z_{0, k}\right\}_{k \geq 1} \subset S$ be with $z_{0, k} \stackrel{k}{\rightarrow} z$. It suffices to show that

$$
\left\|g_{z}-g_{z_{0, k}}\right\|_{\left(\gamma^{\prime}\right)} \stackrel{k}{\rightarrow} 0 \text { as } k \rightarrow \infty .
$$

Let $\left\{z_{1, k}\right\}_{k \geq 1}$ be a subsequence of $\left\{z_{0, k}\right\}_{k \geq 1}$. There exists a bounded interval $J \subset \mathbb{R}$ with

$$
\operatorname{supp}\left(P_{z} \nu\right) \subset J \text { for all } z \in S .
$$

From this, from (4.6), and by Rellich's Theorem (see for instance [Fol, Theorem 9.22]), there exist a subsequence $\left\{z_{2, k}\right\}_{k \geq 1}$ of $\left\{z_{1, k}\right\}_{k \geq 1}$ and $g \in H_{\gamma^{\prime}}(\mathbb{R})$ such that $\left\|g-g_{z_{2, k}}\right\|_{\left(\gamma^{\prime}\right)} \stackrel{k}{\rightarrow} 0$.

As in the proof of the previous lemma, it can be shown that $g=g_{z}$ and so $\left\|g_{z}-g_{z_{2, k}}\right\|_{\left(\gamma^{\prime}\right)} \stackrel{k}{\rightarrow} 0$. Since $\left\{z_{1, k}\right\}_{k \geq 1}$ was an arbitrary subsequence of $\left\{z_{0, k}\right\}_{k \geq 1}$ this gives (4.7) and completes the proof of the lemma.

Proof of part (2) of Theorems 3.1 and 3.7. Let $\lambda \in \mathbb{D} \backslash \mathcal{E}$ be with $\arg \lambda \notin \pi \mathbb{Q}$, $\mathbf{a} \in \mathbb{C}^{\Lambda}$ such that $\mathcal{F}_{\lambda, \mathbf{a}}$ satisfies the SSC, and $p \in \mathbb{P}_{\Lambda}$ with $D_{q}\left(\nu_{\lambda, \mathbf{a}}^{p}\right)>1$ for some $1<q<\infty$. Set $\nu=\nu_{\lambda, \mathbf{a}}^{p}$, then by part (1) of Proposition 4.3 it follows that $P_{z} \nu \in L^{q}(\mathbb{R})$ for all $z \in S$. From this and Proposition 4.7 we get that $\left\{P_{z} \nu\right\}_{z \in S}$ is 
a bounded subset of $\left(L^{q}(\mathbb{R}),\|\cdot\|_{q}\right)$. By Lemma 4.8 it now follows that $z \rightarrow P_{z} \nu$ is continuous as a map from $S$ to $L^{q}(\mathbb{R})$, with respect to the weak topology of $L^{q}(\mathbb{R})$, which completes the proof of part (2) of Theorem 3.1

Part (2) of Theorem 3.7 follows in a similar manner, except that one needs to use part (2) of Proposition 4.3 and Lemma 4.9.

\section{Proof of part (3) of Theorem 3.1}

Throughout this section fix $\alpha \in S, 0<r<1, \Lambda$ a finite nonempty index set, $\mathbf{a} \in \mathbb{C}^{\Lambda}$, and $p \in \mathbb{P}_{\Lambda}$. Write $\lambda=r \alpha, K=K_{\lambda, \mathbf{a}}, \nu=\nu_{\lambda, \mathbf{a}}^{p}$, and $f_{i}=f_{\lambda, \mathbf{a}}^{i}$ for every $i \in \Lambda$. Assume that $\mathcal{F}_{\lambda, \mathbf{a}}$ satisfies the SSC. Assume further that $P_{z} \nu$ is absolutely continuous for each $z \in S$ and write $g_{z}=\frac{d P_{z} \nu}{d \mathcal{L}}$.

Given $i_{1} \ldots i_{k}=\boldsymbol{i} \in \Lambda^{*}$ recall that

$$
f_{\boldsymbol{i}}=f_{i_{1}} \circ \ldots \circ f_{i_{k}} \text { and } p_{\boldsymbol{i}}=p_{i_{1}} \cdot \ldots \cdot p_{i_{k}}
$$

and set $K_{\boldsymbol{i}}=f_{\boldsymbol{i}}(K)$. For $w \in K$ and $k \geq 1$ denote by $\boldsymbol{i}_{k}(w)$ the unique word of length $k$ over $\Lambda$ with $w \in K_{\boldsymbol{i}_{k}(w)}$. Let $T: K \rightarrow K$ be with $T w=f_{\boldsymbol{i}_{1}(w)}^{-1}(w)$ for $w \in K$. It is easy to verify that the system $(K, T, \nu)$ is measure preserving and ergodic.

Lemma 5.1. Let $z \in S$ and $k \geq 1$, then for $\nu$-a.e. $w \in K$

$$
\nu_{z, w}\left(K_{\boldsymbol{i}_{k}(w)}\right)=\frac{g_{\alpha^{-k} z}\left(P_{\alpha^{-k}} T^{k} w\right)}{g_{z}\left(P_{z} w\right)} \cdot p_{\boldsymbol{i}_{k}(w)} r^{-k} .
$$

Proof. From Lemma 3.3 in $[\mathrm{FH}]$ we get that for each $\eta \in S$ and Borel set $A \subset \mathbb{C}$,

$$
\nu_{\eta, w}(A)=\lim _{\delta \downarrow 0} \frac{\nu\left(P_{\eta}^{-1}\left(B\left(P_{\eta} w, \delta\right)\right) \cap A\right)}{\nu\left(P_{\eta}^{-1}\left(B\left(P_{\eta} w, \delta\right)\right)\right)} \text { for } \nu \text {-a.e. } w \in \mathbb{C} .
$$

By Theorem 2.12 in [M] it follows that for each $\eta \in S$,

$$
g_{\eta}\left(P_{\eta} w\right)=\lim _{\delta \downarrow 0} \frac{P_{\eta} \nu\left(B\left(P_{\eta} w, \delta\right)\right)}{2 \delta} \text { for } \nu \text {-a.e. } w \in \mathbb{C} .
$$

From (5.1) and (5.2) we get that for $\nu$-a.e. $w \in K$,

$$
\begin{aligned}
\nu_{z, w}\left(K_{\boldsymbol{i}_{k}(w)}\right) & =\lim _{\delta \downarrow 0} \frac{\nu\left(K_{\boldsymbol{i}_{k}(w)} \cap P_{z}^{-1}\left(B\left(P_{z} w, \delta\right)\right)\right)}{\nu\left(P_{z}^{-1}\left(B\left(P_{z} w, \delta\right)\right)\right)} \\
& =\lim _{\delta \downarrow 0} \frac{2 \delta}{\nu\left(P_{z}^{-1}\left(B\left(P_{z} w, \delta\right)\right)\right)} \cdot \frac{\nu\left(K_{\boldsymbol{i}_{k}(w)} \cap P_{z}^{-1}\left(B\left(P_{z} w, \delta\right)\right)\right)}{2 \delta} \\
& =g_{z}\left(P_{z} w\right)^{-1} \lim _{\delta \downarrow 0} \frac{\nu\left(K_{\boldsymbol{i}_{k}(w)} \cap P_{z}^{-1}\left(B\left(P_{z} w, \delta\right)\right)\right)}{2 \delta} .
\end{aligned}
$$

For each $\delta>0$ set

$$
F_{\delta}=P_{\alpha^{-k} z}^{-1}\left(B\left(P_{\alpha^{-k} z} f_{\boldsymbol{i}_{k}(w)}^{-1}(w), \delta r^{-k}\right)\right)
$$

and for $\eta \in S$ write $\eta^{\perp}=e^{i \pi / 2} \eta$. We have,

$$
\begin{aligned}
P_{z}^{-1}\left(B\left(P_{z} w, \delta\right)\right) & =w+z^{\perp} \mathbb{R}+B(0, \delta) \\
& =f_{\boldsymbol{i}_{k}(w)} \circ f_{\boldsymbol{i}_{k}(w)}^{-1}\left(w+z^{\perp} \mathbb{R}+B(0, \delta)\right) \\
& =f_{\boldsymbol{i}_{k}(w)}\left(f_{\boldsymbol{i}_{k}(w)}^{-1}(w)+\left(\alpha^{-k} z\right)^{\perp} \mathbb{R}+B\left(0, \delta r^{-k}\right)\right) \\
& =f_{\boldsymbol{i}_{k}(w)}\left(F_{\delta}\right) .
\end{aligned}
$$


From this, from (5.3) and since $\nu$ is self-similar,

$$
\begin{aligned}
\nu_{z, w}\left(K_{\boldsymbol{i}_{k}(w)}\right) & =g_{z}\left(P_{z} w\right)^{-1} \lim _{\delta \downarrow 0} \frac{\nu\left(K_{\boldsymbol{i}_{k}(w)} \cap f_{\boldsymbol{i}_{k}(w)}\left(F_{\delta}\right)\right)}{2 \delta} \\
& =g_{z}\left(P_{z} w\right)^{-1} \lim _{\delta \downarrow 0} \frac{1}{2 \delta} \sum_{\boldsymbol{i} \in \Lambda^{k}} p_{\boldsymbol{i}} \cdot \nu\left(f_{\boldsymbol{i}}^{-1}\left(f_{\boldsymbol{i}_{k}(w)}\left(K \cap F_{\delta}\right)\right)\right) .
\end{aligned}
$$

Since $\mathcal{F}_{\lambda, \mathbf{a}}$ satisfies the SSC,

$$
K \cap f_{\boldsymbol{i}}^{-1}\left(f_{\boldsymbol{i}_{k}(w)}(K)\right)=\emptyset \text { for each } \boldsymbol{i} \in \Lambda^{k} \backslash\left\{\boldsymbol{i}_{k}(w)\right\} .
$$

Hence by (5.4) and (5.2),

$$
\begin{aligned}
\nu_{z, w}\left(K_{\boldsymbol{i}_{k}(w)}\right) & =g_{z}\left(P_{z} w\right)^{-1} \lim _{\delta \downarrow 0} p_{\boldsymbol{i}_{k}(w)} r^{-k} \cdot \frac{\nu\left(K \cap F_{\delta}\right)}{2 \delta r^{-k}} \\
& =g_{z}\left(P_{z} w\right)^{-1} \cdot p_{\boldsymbol{i}_{k}(w)} r^{-k} \cdot g_{\alpha^{-k} z}\left(P_{\alpha^{-k} z} f_{\boldsymbol{i}_{k}(w)}^{-1}(w)\right) \\
& =\frac{g_{\alpha^{-k} z}\left(P_{\alpha^{-k}} T^{k} w\right)}{g_{z}\left(P_{z} w\right)} \cdot p_{\boldsymbol{i}_{k}(w)} r^{-k}
\end{aligned}
$$

which completes the proof of the lemma.

Part (3) of Theorem 3.1 follows directly from part (1) combined with the following proposition.

Proposition 5.2. Let $1<q<\infty$, assume that $g_{z} \in L^{q}(\mathbb{R})$ for all $z \in S$, and that $\arg \lambda \notin \pi \mathbb{Q}$. Then for every $z \in S$ the measure $\nu_{z, w}$ has exact dimension $\operatorname{dim}_{H} \nu-1$ for $\nu$-a.e. $w \in \mathbb{C}$.

Proof. By Proposition 4.7 there exists $C>0$ with $\left\|g_{z}\right\|_{q} \leq C$ for all $z \in S$. Fix $z \in S$, then since $\mathcal{F}_{\lambda, \mathbf{a}}$ satisfies the SSC it suffices to show that for $\nu$-a.e. $w \in K$,

$$
\lim _{k} \frac{\log \nu_{z, w}\left(K_{\boldsymbol{i}_{k}(w)}\right)}{\log r^{k}}=\operatorname{dim}_{H} \nu-1 .
$$

For every $k \geq 1$ set $z_{k}=\alpha^{-k} z$ and

$$
A_{k}=\left\{w \in K: g_{z_{k}}\left(P_{z_{k}} w\right) \geq k^{2 /(q-1)}\right\} .
$$

We have,

$$
\begin{aligned}
\nu\left(A_{k}\right)=P_{z_{k}} \nu\left\{x \in \mathbb{R}:\left(g_{z_{k}}(x)\right)^{q-1}\right. & \left.\geq k^{2}\right\} \\
& \leq \frac{1}{k^{2}} \cdot \int\left(g_{z_{k}}(x)\right)^{q-1} d P_{z_{k}} \nu(x)=\frac{\left\|g_{z_{k}}\right\|_{q}^{q}}{k^{2}} \leq \frac{C^{q}}{k^{2}} .
\end{aligned}
$$

From this and since $(K, T, \nu)$ is measure preserving,

$$
\sum_{k=1}^{\infty} \nu\left(T^{-k}\left(A_{k}\right)\right)=\sum_{k=1}^{\infty} \nu\left(A_{k}\right)<\infty .
$$

Now from the Borel-Cantelli lemma it follows that for $\nu$-a.e. $w \in K$,

$$
g_{z_{k}}\left(P_{z_{k}} T^{k} w\right) \leq k^{2 /(q-1)} \text { for all large enough } k \geq 1 .
$$

For $k \geq 1$ set

$$
B_{k}=\left\{w \in K: g_{z_{k}}\left(P_{z_{k}} w\right) \leq k^{-2}\right\}
$$


and let $J \subset \mathbb{R}$ be a bounded interval with $\operatorname{supp}\left(P_{\eta} \nu\right) \subset J$ for all $\eta \in S$. We have,

$$
\begin{aligned}
\nu\left(B_{k}\right) & =P_{z_{k}} \nu\left\{x \in \mathbb{R}: g_{z_{k}}(x) \leq k^{-2}\right\} \\
& =\int_{J} 1_{\left\{g_{z_{k}}(x) \leq k^{-2}\right\}} g_{z_{k}}(x) d x \leq \frac{\mathcal{L}(J)}{k^{2}},
\end{aligned}
$$

and so $\sum_{k=1}^{\infty} \nu\left(T^{-k}\left(B_{k}\right)\right)<\infty$. From (5.5) and the Borel-Cantelli lemma it now follows that for $\nu$-a.e. $w \in K$,

$$
k^{-2} \leq g_{z_{k}}\left(P_{z_{k}} T^{k} w\right) \leq k^{2 /(q-1)} \text { for all large enough } k \geq 1 .
$$

Denote by $H(p)$ the entropy of $p$, then $\operatorname{dim}_{H} \nu=\frac{H(p)}{-\log r}$ by (2.4). From the Shannon-McMillan-Breiman theorem, applied to the ergodic system $(K, T, \nu)$ and partition $\left\{f_{i}(K)\right\}_{i \in \Lambda}$, it follows that for $\nu$-a.e. $w \in K$,

$$
\lim _{k}-\frac{\log p_{\boldsymbol{i}_{k}(w)}}{k}=\lim _{k}-\frac{\log \nu\left(K_{\boldsymbol{i}_{k}(w)}\right)}{k}=H(p) .
$$

From This, Lemma 5.1, and (5.6), we get that for $\nu$-a.e. $w \in K$

$$
\begin{aligned}
\lim _{k} \frac{\log \nu_{z, w}\left(K_{\boldsymbol{i}_{k}(w)}\right)}{\log r^{k}} & =\lim _{k} \frac{1}{k \log r} \cdot \log \left(\frac{g_{z_{k}}\left(P_{z_{k}} T^{k} w\right)}{g_{z}\left(P_{z} w\right)} \cdot p_{\boldsymbol{i}_{k}(w)} r^{-k}\right) \\
& =\lim _{k} \frac{1}{k \log r} \cdot \log \left(p_{\boldsymbol{i}_{k}(w)} r^{-k}\right)=\frac{H(p)}{-\log r}-1=\operatorname{dim}_{H} \mu-1,
\end{aligned}
$$

which completes the proof of the proposition.

\section{Proof of Corollary 3.4}

Proof of Corollary 3.4. Let $\lambda \in \mathbb{D} \backslash \mathcal{E}$ be with $\arg \lambda \notin \pi \mathbb{Q}, \Lambda$ a finite nonempty set, and $\mathbf{a} \in \mathbb{C}^{\Lambda}$ such that $\mathcal{F}_{\lambda, \mathbf{a}}$ satisfies the SSC. Set $K=K_{\lambda, \mathbf{a}}$, let $s=\operatorname{dim}_{H} K$ and assume $s>1$. Recall that for $z \in S$ and $x \in \mathbb{R}$ we write $K_{z, x}=K \cap P_{z}^{-1}\{x\}$.

Let $\left(p_{i}\right)_{i \in \Lambda}=p \in \mathbb{P}_{\Lambda}$ be with $p_{i}=|\Lambda|^{-1}$ for each $i \in \Lambda$, and set $\nu=\nu_{\lambda, \mathbf{a}}^{p}$. Note that $\nu=\left.C_{0} \cdot \mathcal{H}^{s}\right|_{K}$ for some normalizing constant $0<C_{0}<\infty$. By (2.3) and (2.5),

$$
D_{2}(\nu)=\frac{\log \|p\|_{2}^{2}}{\log |\lambda|}=\frac{\log |\Lambda|^{-1}}{\log |\lambda|}=\operatorname{dim}_{H} K>1 .
$$

Hence, by part (1) of Theorem 3.1 and Proposition 4.7, it follows that $\left\{P_{z} \nu\right\}_{z \in S}$ is a Bounded subset of $\left(L^{2}(\mathbb{R}),\|\cdot\|_{2}\right)$. Let $C>0$ be with $\left\|g_{z}\right\|_{2} \leq C$ for all $z \in S$, where $g_{z}=\frac{d P_{z} \nu}{d \mathcal{L}}$.

Fix $z \in S$ and set

$$
B=\left\{w \in K: \operatorname{dim}_{H} K_{z, P_{z} w}=s-1\right\} .
$$

By part (3) of Theorem 3.1.

$$
\operatorname{dim}_{H} \nu_{z, w}=\operatorname{dim}_{H} \nu-1=s-1 \text { for } \nu \text {-a.e. } w \in K .
$$

Additionally, $\nu_{z, w}$ is supported on $K_{z, P_{z} w}$ for $\nu$-a.e. $w \in K$. This shows that $\operatorname{dim}_{H} K_{z, P_{z} w} \geq s-1$ for $\nu$-a.e. $w \in K$. From Theorem 5.8 in Fal1 it follows that $\operatorname{dim}_{H}\left(K_{z, x}\right) \leq s-1$ for $\mathcal{L}$-a.e. $x \in \mathbb{R}$. Since $P_{z} \nu \ll \mathcal{L}$ this shows that $\operatorname{dim}_{H} K_{z, P_{z} w} \leq s-1$ for $\nu$-a.e. $w \in K$, which gives $\nu(B)=1$.

Write

$$
A=\left\{w \in K: \mathcal{P}^{s-1}\left(K_{z, P_{z} w}\right)>0\right\},
$$


and recall the map $T: K \rightarrow K$ from the previous section. Let $\alpha \in S$ and $0<r<1$ be with $\lambda=r \alpha$, and for each $k \geq 1$ set $z_{k}=\alpha^{-k} z$. Fix $n \geq 1$, for each $k \geq 1$ let

$$
A_{n, k}=\left\{w \in K: g_{z_{k}}\left(P_{z_{k}} T^{k} w\right) \leq n\right\},
$$

and write

$$
A_{n}=\cap_{N \geq 1} \cup_{k \geq N} A_{n, k} .
$$

Since $(K, T, \nu)$ is measure preserving,

$$
\begin{aligned}
\nu\left(K \backslash A_{n, k}\right) & =P_{z_{k}} \nu\left\{x \in \mathbb{R}: g_{z_{k}}(x)>n\right\} \\
& \leq \frac{1}{n} \int g_{z_{k}}(x) d P_{z_{k}} \nu(x)=\frac{\left\|g_{z_{k}}\right\|_{2}^{2}}{n} \leq \frac{C^{2}}{n} .
\end{aligned}
$$

Hence,

Write

$$
\nu\left(A_{n}\right)=\lim _{N} \nu\left(\cup_{k \geq N} A_{n, k}\right) \geq \liminf _{N} \nu\left(A_{n, N}\right) \geq 1-\frac{C^{2}}{n} .
$$

$$
D=\left\{w \in K: \nu_{z, w}\left(A_{n}\right)>0\right\}
$$

then $\nu(D) \geq 1-\frac{C^{2}}{n}$.

For $k \geq 1$ and $w \in K$ let $K_{i_{k}(w)}$ be as defined in the previous section. From Lemma 5.1 we get that for $\nu$-a.e. $w \in K$ and $\nu_{z, w}$-a.e. $\eta \in A_{n}$,

$$
\begin{aligned}
\liminf _{k \rightarrow \infty} \frac{\nu_{z, w}\left(K_{\boldsymbol{i}_{k}(\eta)}\right)}{|\lambda|^{k(s-1)}} & =\liminf _{k \rightarrow \infty} \frac{\nu_{z, \eta}\left(K_{\boldsymbol{i}_{k}(\eta)}\right)}{|\lambda|^{k(s-1)}} \\
& =\liminf _{k \rightarrow \infty} \frac{g_{z_{k}}\left(P_{z_{k}} T^{k} \eta\right) \cdot|\Lambda|^{-k}|\lambda|^{-k}}{g_{z}\left(P_{z} \eta\right)|\lambda|^{k(s-1)}} \\
& \leq \frac{n}{g_{z}\left(P_{z} \eta\right)} \lim _{k \rightarrow \infty} \frac{|\Lambda|^{-k}}{|\lambda|^{k s}}=\frac{n}{g_{z}\left(P_{z} w\right)}
\end{aligned}
$$

where in the last equality we have used $s=\frac{\log |\Lambda|}{-\log |\lambda|}$. Now since $\mathcal{F}_{\lambda, \mathbf{a}}$ satisfies the SSC, we get that for $\nu$-a.e. $w \in K$ there exists $0<C_{w, n}<\infty$ with,

$$
\liminf _{\delta \downarrow 0} \frac{\nu_{z, w}(B(\eta, \delta))}{(2 \delta)^{s-1}} \leq C_{w, n} \text { for } \nu_{z, w} \text {-a.e. } \eta \in A_{n} .
$$

From this and by Theorem 6.11 in $[\mathrm{M}]$ it follows that for $\nu$-a.e. $w \in D$,

$$
\mathcal{P}^{s-1}\left(K_{z, P_{z} w}\right) \geq C_{w, n}^{-1} \cdot \nu_{z, w}\left(A_{n}\right)>0,
$$

and so $w \in A$. This gives $\nu(A) \geq \nu(D) \geq 1-\frac{C^{2}}{n}$, which shows $\nu(A)=1$ since $n \geq 1$ can be taken to be arbitrarily large. We have thus shown $\nu(A \cap B)=1$. From this and since the measures $\nu$ and $\left.\mathcal{H}^{s}\right|_{K}$ are equivalent, we obtain part (11) of the corollary.

Write,

$$
F=\left\{x \in \mathbb{R}: \operatorname{dim}_{H}\left(K_{z, x}\right)=s-1 \text { and } \mathcal{P}^{s-1}\left(K_{z, x}\right)>0\right\} .
$$

From $\nu(A \cap B)=1$ we get $P_{z} \nu(F)=1$. Hence by the Cauchy-Schwarz inequality,

$$
1=P_{z} \nu(F)=\int 1_{F}(x) g_{z}(x) d x \leq\left\|1_{F}\right\|_{2} \cdot\left\|g_{z}\right\|_{2} \leq C\left\|1_{F}\right\|_{2},
$$

which gives $\mathcal{L}(F) \geq C^{-2}$. This completes the proof of part (2) of the corollary with $c=C^{-2}$. 


\section{Proof of part (44) of Theorem 3.1}

The following proof is an adaptation of the argument in [PSS, Proposition 3.1].

Proof of part (4) of Theorem 3.1. Let $\lambda \in \mathbb{D} \backslash \mathcal{E}$ be with $\arg \lambda \notin \pi \mathbb{Q}, \Lambda$ a finite nonempty set, $\mathbf{a} \in \mathbb{C}^{\Lambda}$ such that $\mathcal{F}_{\lambda, \mathbf{a}}$ satisfies the SSC, and $p \in \mathbb{P}_{\Lambda}$ with $D_{q}\left(\nu_{\lambda, \mathbf{a}}^{p}\right)>$ 1 for some $1<q<\infty$. Set $K=K_{\lambda, \mathbf{a}}, \nu=\nu_{\lambda, \mathbf{a}}^{p}$, and $f_{i}=f_{\lambda, \mathbf{a}}^{i}$ for $i \in \Lambda$. By part (11) of Theorem 3.1 it follows $P_{z} \nu \in L^{q}(\mathbb{R})$ for each $z \in S$. Hence it suffices to show that $\left.\mathcal{L}\right|_{P_{z}(K)} \ll P_{z} \nu$ for each $z \in S$.

By Proposition 4.7 there exists $C>0$ with $\left\|g_{z}\right\|_{q} \leq C$ for all $z \in S$, where $g_{z}=\frac{d P_{z} \nu}{d \mathcal{L}}$. From

$$
\operatorname{dim}_{H} K \geq \operatorname{dim}_{H} \nu \geq D_{q}(\nu)>1
$$

and part (2) of Corollary 3.4, it follows that there exists $c>0$ with

$$
\mathcal{L}\left(P_{z}(K)\right)>c \text { for all } z \in S .
$$

Write,

$$
\beta=\sup \left\{\frac{\mathcal{L}(A)}{\mathcal{L}\left(P_{z}(K)\right)}: z \in S, A \subset P_{z}(K) \text { is Borel, and } P_{z} \nu(A)=0\right\} .
$$

Assume by contradiction that $\beta=1$. Given $\epsilon>0$ there exists $z \in S$ and a Borel set $A \subset P_{z}(K)$ with $P_{z} \nu(A)=0$ and $\frac{\mathcal{L}(A)}{\mathcal{L}\left(P_{z}(K)\right)}>1-\epsilon$. We have

$$
\mathcal{L}\left(P_{z}(K) \backslash A\right)<\epsilon \cdot \mathcal{L}\left(P_{z}(K)\right),
$$

hence by Hölder's inequality,

$$
\begin{aligned}
1 & =P_{z} \nu\left(P_{z}(K) \backslash A\right)=\int_{P_{z}(K) \backslash A} g_{z} d \mathcal{L} \\
& \leq\left\|g_{z}\right\|_{q} \cdot\left(\mathcal{L}\left(P_{z}(K) \backslash A\right)\right)^{1-q^{-1}}<C \cdot\left(\epsilon \cdot \mathcal{L}\left(P_{z}(K)\right)\right)^{1-q^{-1}} .
\end{aligned}
$$

For a sufficiently small $\epsilon>0$ this clearly yields a contradiction, and so we must have $\beta<1$.

Fix $z \in S$ and let $A_{0} \subset P_{z}(K)$ be a Borel set with $P_{z} \nu\left(A_{0}\right)=0$. It suffices to prove that $\mathcal{L}\left(A_{0}\right)=0$. We will show that for each $x \in \mathbb{R}$

$$
\limsup _{\delta \downarrow 0} \frac{\mathcal{L}\left((x-\delta, x+\delta) \cap A_{0}\right)}{2 \delta}<1,
$$

and so that $A_{0}$ has no Lebesgue density points. From this and Lebesgue's density theorem it follows that we must have $\mathcal{L}\left(A_{0}\right)=0$. If $x \notin P_{z}(K)$ then $(x-\delta, x+\delta) \cap$ $A_{0}=\emptyset$ for all sufficiently small $\delta>0$, and so (7.2) is clearly satisfied.

Fix $x \in P_{z}(K)$ and $0<\delta<\frac{c}{2}$. By (7.1) there exists $m \geq 1$ and $i_{1} \ldots i_{m}=\boldsymbol{i} \in \Lambda^{m}$ such that $x \in P_{z} f_{\boldsymbol{i}}(K) \subset(x-\delta, x+\delta)$ and

$$
P_{z} f_{i_{1} \ldots i_{m-1}}(K) \nsubseteq(x-\delta, x+\delta),
$$

where recall that $f_{i}=f_{i_{1}} \circ \ldots \circ f_{i_{m}}$. Let $\alpha \in S$ and $0<r<1$ be with $\lambda=r \alpha$ and set $a=-P_{z} f_{\boldsymbol{i}}(0)$. Then

$$
P_{z} f_{i}=\tau_{a} M_{r^{m}} P_{\alpha^{-m} z}
$$

where recall that $\tau_{a}(y)=y-a$ and $M_{r^{m}}(y)=r^{m} y$ for $y \in \mathbb{R}$. By (7.3),

$$
r^{m} \cdot \operatorname{diam}(K)=r \cdot \operatorname{diam}\left(f_{i_{1} \ldots i_{m-1}}(K)\right) \geq r \delta \text {. }
$$


From this, (7.4), and (7.1),

$$
\mathcal{L}\left(P_{z} f_{\boldsymbol{i}}(K)\right)=r^{m} \cdot \mathcal{L}\left(P_{\alpha^{-m} z}(K)\right)>\frac{r \delta c}{\operatorname{diam}(K)}=b \delta,
$$

with $b=\frac{r c}{\operatorname{diam}(K)}$.

Since $\nu$ is self-similar we have $P_{z} f_{\boldsymbol{i}} \nu \ll P_{z} \nu$. From this and since $P_{z} \nu\left(A_{0} \cap\right.$ $\left.P_{z} f_{\boldsymbol{i}}(K)\right)=0$,

$$
\begin{array}{r}
0=P_{z} f_{\boldsymbol{i}} \nu\left(A_{0} \cap P_{z} f_{\boldsymbol{i}}(K)\right)=\tau_{a} M_{r^{m}} P_{\alpha^{-m} z} \nu\left(A_{0} \cap \tau_{a} M_{r^{m}} P_{\alpha^{-m} z}(K)\right) \\
\quad=P_{\alpha^{-m} z} \nu\left(M_{r^{-m}} \tau_{-a}\left(A_{0}\right) \cap P_{\alpha^{-m} z}(K)\right) .
\end{array}
$$

Now by the definition of $\beta$,

$$
\mathcal{L}\left(M_{r^{-m}} \tau_{-a}\left(A_{0}\right) \cap P_{\alpha^{-m} z}(K)\right) \leq \beta \cdot \mathcal{L}\left(P_{\alpha^{-m} z}(K)\right) .
$$

Hence,

$$
\mathcal{L}\left(A_{0} \cap \tau_{a} M_{r^{m}} P_{\alpha^{-m} z}(K)\right) \leq \beta \cdot \mathcal{L}\left(\tau_{a} M_{r^{m}} P_{\alpha^{-m} z}(K)\right),
$$

which gives,

$$
\mathcal{L}\left(A_{0} \cap P_{z} f_{\boldsymbol{i}}(K)\right) \leq \beta \cdot \mathcal{L}\left(P_{z} f_{\boldsymbol{i}}(K)\right) .
$$

From this and (7.5) we get,

$$
\begin{aligned}
\mathcal{L}\left((x-\delta, x+\delta) \backslash A_{0}\right) & \geq \mathcal{L}\left(P_{z} f_{\boldsymbol{i}}(K) \backslash A_{0}\right) \\
& =\mathcal{L}\left(P_{z} f_{\boldsymbol{i}}(K)\right)-\mathcal{L}\left(P_{z} f_{\boldsymbol{i}}(K) \cap A_{0}\right) \\
& \geq(1-\beta) \mathcal{L}\left(P_{z} f_{\boldsymbol{i}}(K)\right) \geq(1-\beta) b \delta .
\end{aligned}
$$

Since $(1-\beta) b>0$ does not depend on $\delta$ this gives (7.2), which completes the proof.

\section{REFERENCES}

[EW] M.Einsiedler and T.Ward, Ergodic Theory with a View Towards Number Theory (Graduate Texts in Mathematics, 259). Springer, London, 2011.

[Fal1] K. Falconer, The geometry of fractal sets. Cambridge Univ. Press, New York, 1985.

[Fal2] K. Falconer, Techniques in fractal geometry. John Wiley \& Sons, 1997.

[FJ1] K. Falconer and X. Jin, Exact dimensionality and projections of random self-similar measures and sets. J. Lond. Math. Soc. (2), 90(2) (2014), 388-412.

[FJ2] K. Falconer and X. Jin, Dimension conservation for self-similar sets and fractal percolation. Int. Math. Res. Not. 2015(24) (2015), 13260-13289.

[FLR] A.-H. Fan, K.-S. Lau, and H. Rao, Relationships between different dimensions of a measure, Monatsh. Math. 135 (2002), 191-201.

[FH] D.-J. Feng and H. Hu, Dimension theory of iterated function systems. Comm. Pure Appl. Math. 62 (2009), 1435-1500.

[Fol] G. Folland, Real analysis: Modern techniques and their applications. Pure and Applied Mathematics. Wiley-Interscience, second edition, 1999.

[Fur] H.Furstenberg, Ergodic fractal measures and dimension conservation. Ergodic Theory Dynam. Systems 28 (2008), 405-422.

[HS] M. Hochman and P. Shmerkin, Local entropy averages and projections of fractal measures. Ann. of Math. 175(3) (2012), 1001-1059.

[HK] B. Hunt and V. Kaloshin, How projections affect the dimension spectrum of fractal measures. Nonlinearity 10 (1997), 1031-1046.

[JM] M. Jarvenpaa and P. Mattila, Hausdorff and packing dimensions and sections of measures. Mathematika 45 (1998), 55-77.

[M] P. Mattila, Geometry of sets and measures in euclidean spaces. Fractals and rectifiability. Cambridge Studies in Advanced Mathematics, 44. Cambridge University Press, Cambridge, 1995. 
[MS] D. Mauldin and K. Simon, The equivalence of some Bernoulli convolutions to Lebesgue measure. Proc. Amer. Math. Soc. 126, no. 9 (1998), 2733-2736.

[PSS] Y. Peres, W. Schlag, and B. Solomyak, Sixty years of Bernoulli convolutions. In Fractal geometry and stochastics II, pages 39-65. Springer, 2000.

[PS] Y. Peres and P. Shmerkin, Resonance between cantor sets. Ergodic Theory and Dynamical Systems 29 (2009), 201-221.

[Rap1] A. Rapaport, On the Hausdorff and packing measures of slices of dynamically defined sets. J. Frac. Geom. 3(1) (2016), 33-74.

[Rap2] A. Rapaport, A self-similar measure with dense rotations, singular projections and discrete slices. Adv. in Math. 321 (2017), 529-546.

[Sh1] P. Shmerkin, On the exceptional set for absolute continuity of Bernoulli convolutions. Geom. Funct. Anal. 24(3) (2014), 946-958.

[Sh2] P. Shmerkin, On Furstenberg's intersection conjecture, self-similar measures, and the $L^{q}$ norms of convolutions. Preprint, arXiv:1609.07802 2016.

[SS1] P. Shmerkin and B. Solomyak, Absolute continuity of self-similar measures, their projections and convolutions. Trans. Amer. Math. Soc. 368(7) (2016), 5125-5151.

[SS2] P. Shmerkin and B. Solomyak, Absolute continuity of complex Bernoulli convolutions. Math. Proc. Cambridge Philos. Soc. 161(3) (2016), 435-453.

Centre for Mathematical Sciences, Wilberforce Road, Cambridge CB3 0WA, UK

E-mail: ariel.rapaport@mail.huji.ac.il 